\title{
Una propuesta de nivel cero en la didáctica de los Estudios Franceses desde las TIC
}

\author{
A cero level proposal in the didactic \\ of French studies from ITC
}

\author{
Esmeralda Vicente Castañares \\ Universidad de Extremadura \\ vicent@unex.es
}

\begin{abstract}
The tearing easiness of access to any web content, as well as its huge quantity, make complex the application of a cero level quality informative filter. We consider this ICT screening should be done whenever students are going to use it for their own researching tasks in French Studies. This study's essential target would like to focus on the students' acquisition of guide ICT tools, which could help them to develop or increase a critical and selective thinking. This would enable them to take suitable decisions whenever making a choice and using ICT in a responsible, sure, collaborative, cooperative way. In order to attain these goals, we would start from a previous survey among students, with the aim to compile their possible knowledge concerning these "guide ICT tools" (example: Agencia Española de Protección de Datos' web and its "Lista Robinson").
\end{abstract}

\section{Key-words}

Data protection, screening, security, cooperation, ICT.

\section{Résumé}

La vertigineuse facilité d'accès à n'importe quel contenu web, ainsi que son imposante quantité, entravent la réalisation d'un tri de qualité informative au niveau zéro. On estime impératif ce filtrage TICE, au moment d'être utilisé par les étudiants pour leurs propres travails de recherche en Études Françaises. L'objectif fondamental de cette étude serait de réussir à ce que les étudiants disposent des outils TICE guide qui puissent les aider à développer ou fomenter un esprit critique et sélectif. Celui-ci leur permettrait de prendre des décisions adéquates au moment de sélectionner et utiliser les TICE de manière responsable, sure, collaborative et coopérative. Pour atteindre ces objectifs on partira d'un sondage préalable entre les étudiants, à l'objet de compiler leur possible connaissance de ces "outils guide" (exemple: web de la Agencia Española de Protección de Datos et sa "Lista Robinson"). Dorénavant, on agira à une analyse systématique de chaque contenu TICE utilisé qui puisse fournir cette capacité sélective aux étudiants.

\section{Mots-clés}

Protection des données, filtrage, sécurité, coopération, TICE. 
Anales de Filología Francesa, n. ${ }^{\circ}$ 29, 2021

UNA PROPUESTA DE NIVEL CERO EN LA DIDÁCTICA DE LOS ESTUDIOS FRANCESES DESDE LAS TIC

\section{Introducción}

Estamos en un mundo global, la pandemia que hemos y estamos sufriendo así nos lo demuestra. De igual modo, la red era, en nuestros días - hasta el protagonismo cobrado por el Covid-19- el mayor ejemplo de esa globalización. Vivir en un mundo interconectado tiene sus ventajas e inconvenientes. En el ámbito universitario, tal como se viene haciendo ya desde la enseñanza obligatoria, la implantación de aulas TIC es un hecho. En esta investigación queremos poner de manifiesto la relevancia de ambas caras de la aplicación transversal de las TIC a otras áreas de estudio no específicamente relacionadas con la tecnología. Por ello, siguiendo en todo momento la planificación establecida en la materia que relaciona Estudios Franceses y TIC, en la que proponemos introducir el estudio experimental que planteamos, con esta Propuesta, trataremos de mostrar a nuestro alumnado los pros y los contras del uso de las TIC e Internet. Dentro de los pros, encontramos el gran abanico de posibilidades que las TIC ofrecen en el aprendizaje de las lenguas y culturas. Sin embargo, entre los contras, tenemos el mal uso de la red por parte de ciertas instituciones, empresas, publicidad, ciberacosadores, hackers... que se ha visto acrecentado en estos últimos tiempos en que la educación, la economía, la salud, y todos y cada uno de los ámbitos de la vida del planeta han sido dependientes en grado sumo de la tecnología. Nuevamente, dentro de los pros, nosotros aportamos en esta Propuesta una muestra de la cantidad y posibilidad de "antídotos" válidos contra estos usos probablemente poco éticos de la red y las tecnologías: el acoso publicitario, el ciberacoso por parte de personas anónimas, entidades, robo de datos por parte de hackers, recopilación de datos por entidades oficiales que pueden ser ofrecidos a terceros, etc. Entre los recursos para paliar estos usos poco éticos encontramos, verbigracia, webs colaborativas promocionales del cooperativismo, la igualdad y la no violencia.

La lectura del "Informe de iniciativas y herramientas de evaluación online universitaria en el contexto del COVID-19" (González, Marco y Medina, 2020) del Ministerio de Universidades español, nos ha alertado sobre la posible sobreexposición de datos a la que podría aventurarse nuestro alumnado, al utilizar las TIC e Internet para sus trabajos de investigación en Estudios Franceses. Concretamente, en este informe, nos ha llamado la atención de manera exponencial el punto "2.2. Contexto angloamericano. Estados Unidos". En el caso paradigmático de Estados Unidos no nos sorprende su positiva generalización del uso de las TIC en buena parte de los Estados; ya implantado mucho antes de esta pandemia. Por un lado, al ser Estados Unidos un país tan tremendamente extenso, entendemos que estas herramientas se hacen cuando menos necesarias. A esta cuestión habría que añadir el hecho de que buena parte de las empresas punteras a nivel tecnológico mundial proceden de este territorio. Es, por tanto, lo que cabría esperar. Por otro lado, encontramos contraproducente el uso de datos personales por parte de plata- 
formas y programas elaborados por estas mismas empresas. Del mismo modo, nos cuesta entender que el mercantilismo impere por encima de los valores educativos. Todo ello, pone en riesgo la privacidad de alumnos y profesores, por lo que sus métodos no nos parecen rigurosos ni dignos de seguir:

Con respecto a los cambios en la evaluación, la supervisión de exámenes online es una herramienta incorporada desde hace años por un gran número de universidades [...] para mantener la seguridad a distancia. Muchos de los cursos que se imparten usando plataformas tipo Blackboard ya están configurados para usar programas de vigilancia ("proctoring") como Proctortrack [...], TopHat[...], ProctorU[...], Proctorio[...], o Examinity[...], entre los más extendidos. Estos sistemas controlan, a través de la webcam del estudiante, sus movimientos oculares, niveles de audio en su entorno, las aplicaciones o procesos en ejecución durante la sesión, reconocimiento facial de la tarjeta de identificación o el ritmo de las teclas. La crisis de la Covid-19 ha incrementado su implantación y su uso, aunque algunos de ellos se consideran invasivos (asignan "niveles de sospecha" según el patrón de movimiento durante la grabación) o conservan derechos sobre los datos que graban y que pueden compartir con terceros. (González, Marco y Medina, 2020: 9)

\title{
2. Presentación de la Propuesta
}

La Propuesta que aquí presentamos es una experimentación llevada a cabo por nosotros, con 6 alumnos de $2^{\circ}$ curso del Grado en Lenguas Modernas y Literaturas comparadas-Francés de una universidad española. Incluimos esta Propuesta para ser aplicada como premisa a llevar a cabo dentro del seguimiento de contenidos, competencias, objetivos y evaluación de una materia que combina los Estudios Franceses y las TIC. Queremos insistir en el hecho de que se trata de un estudio experimental, que ofrecemos generosamente en abierto, de modo que otros profesionales que quieran, puedan llevarlo a cabo en sus aulas, mejorarlo, probablemente, por qué no. Ya que, si aplicamos la reflexión sobre qué es enseñar, de Magdalena Arillo Crespo:

\begin{abstract}
Enseñar, como aprender es una espiral; no importa dónde empieces, siempre seguirá el ciclo de planificación, acción/observación y evaluación. Sólo nos tenemos que asegurar, una vez más, de no priorizar una y olvidar otra. Si erramos al planificar, siempre que observemos y anotemos lo que realmente sucede en el aula, podremos valorar lo positivo, para re-usarlo en el futuro, y tomar medidas correctoras sobre lo que no funcionó, así como incluir todo aquello que sucedió y excedió a nuestra planificación para valorarlo como aprendizaje. (Arillo Crespo, 1992: 56-57)
\end{abstract}

De tal modo, quisiéramos advertir a aquellos lectores e investigadores que esperen encontrar en este estudio unos resultados inmediatos de objetivos alcanzados. Nosotros no hemos entendido ni planificado este estudio como una fórmula matemática que tenga el 
deber de ofrecer resultados inmediatos. El título de nuestro artículo lo indica con claridad: una Propuesta a nivel cero... Es decir, una propuesta de la que partir, un acercamiento a los Estudios Franceses desde las TIC, en el que cada contenido web que se consulte, se haga con los conocimientos de ciberseguridad básicos para no ser víctima de acoso publicitario, ciberacoso, robo de datos, identidad; y ser, a su vez, un usuario responsable al hacer uso de las herramientas TIC enfocadas a los Estudios Franceses. Desde la planificación de esta Propuesta, hemos sido totalmente conscientes de que no podríamos obtener grandes objetivos tan a corto plazo. Por tal motivo, nuestra planificación ha enfocado la consecución de objetivos más bien a lo largo de los estudios del Grado en Lenguas Modernas y Literaturas Comparadas - Francés. Somos conscientes de que, como docentes responsables, no podemos ni debemos obligar a nuestros alumnos a examinar siempre las cookies y políticas de privacidad de cualquier web que consulten, al recabar información para sus trabajos de Estudios Franceses, como herramientas básicas de ciberseguridad. En consecuencia, hemos procedido, únicamente, a poner de manifiesto la existencia de dichas herramientas. Les hemos hecho saber que pueden usarlas, que sabemos que revisarlas supone una mayor dedicación temporal en la realización de cualquier trabajo académico. Sin embargo, éstas, utilizadas de manera sistemática, podrían proporcionar una coraza protectora presente y futura a los usuarios que quisieran aprovecharlas. La Propuesta se planifica para ser puesta en marcha en $1^{\circ}$ o $2^{\circ}$ curso del mencionado Grado. De tal modo, se ofrece la posibilidad de poder hacer un seguimiento de esta Propuesta en futuras materias con el grupo estudiado. En virtud de ello, no nos interesan únicamente los objetivos cortoplacistas puesto que no serían realistas ni fáciles de obtener. No quiere ello decir que no tengamos expectativas de conseguirlos.

Como veremos a continuación, si bien el número de alumnos que han participado en este ensayo es reducido, sus características presentan un nutrido ejemplo de diversidad geográfica, personal, académica y profesional. Esta diversidad contribuirá al enriquecimiento de este estudio. De igual manera, la movilidad académica internacional que algunos de ellos van a llevar a cabo, en breve, al sistema cultural francés, nos permitirá observar si ponen en práctica la experiencia que han iniciado en y desde el sistema cultural español, en su inmersión directa en el sistema cultural francés. Esta Propuesta es, por tanto, una propuesta viva, que pretendemos no se cierna únicamente a los moldes de un cuatrimestre del curso de una titulación. Nuestra intención es que sus mejores frutos puedan recogerse a medio y largo plazo. El hecho de que haya sido puesta en práctica en el $2^{\circ}$ curso del Grado, será un agente facilitador de dicho seguimiento de los objetivos de mayor calado en los cursos $3^{\circ}$ y $4^{\circ}$. Los alumnos que siguen el próximo curso en España, podrán hacer uso de las herramientas que les hemos proporcionado, de las que ellos mismos han compartido con el resto en sus trabajos colaborativos y cooperativos desde aquí. Los alumnos que parten como Erasmus a sumergirse en la lengua y cultura francesas, 
en suelo galo, tendrán así mismo una gran oportunidad para hacer uso de esas mismas herramientas. Probablemente sea allí, sobre el terreno, cuando descubran con mayor profundidad, la utilidad de las mismas. De igual modo, dentro de los objetivos a medio y largo plazo esperados, contamos con que se produzca una contaminación positiva de los alumnos que parten fuera; de suerte que su experiencia de intercambio anime al resto del grupo que se queda el siguiente curso en España, para que en futuros cursos decidan por sí mismos salir a experimentar de manera similar, sobre el terreno, las herramientas con las que han trabajado en esta Propuesta.

\section{Características del grupo académico participante en el ensayo}

Los alumnos que han participado en este estudio proceden en su mayoría de pueblos de provincias. El grupo que ha asistido a clase consta de 3 alumnas y 3 alumnos, respectivamente. De entre ellos, uno aparece intermitentemente, y solamente hemos podido recabar datos de su participación en un primer cuestionario que adjuntamos al final del artículo. Aun así, nosotros hemos querido tener en cuenta su testimonio, ya que consideramos que esta persona, de 25 años, unos cuantos años mayor que el resto, podría ofrecer tanto a sus compañeros como a nosotros mismos, otra perspectiva de este estudio. Nuestros/as alumnos/as son personas bastante activas, dado que todos ellos han realizado o realizan algún trabajo que compaginan con los estudios, en empresas familiares, trabajos de verano tanto en España como en Francia, como au pairs o bien en el sector turístico. Alguno de ellos cuenta ya con una titulación anterior. En lo referente a sus expectativas laborales tras el Grado, unos enfocan ya sus pasos hacia la traducción profesional, otros tienen intención de dedicarse a la enseñanza y al turismo. Dos de nuestros alumnos han solicitado y les ha sido concedida una movilidad Erasmus a una universidad francesa. Uno de ellos ha estado previamente de intercambio en Francia, experiencia de la que dice guardar buenos recuerdos; lo que le lleva a no dudar en solicitar esta movilidad Erasmus. Excepto el caso excepcional del alumno que ha asistido intermitentemente, anteriormente comentado, el resto de la clase asiste con asiduidad. El alumnado presenta gran interés por la combinación que ofrece la materia entre conocimiento, manejo de TIC y aplicación a la vida cotidiana y profesional en relación con los E.F. Todos nuestros alumnos, pertenecientes a la Generación Z, hacen un uso cotidiano de redes sociales, de Internet, y tienen sus propios influencers de referencia. Generalmente, no ven la televisión ni escuchan la radio. En el caso de la radio, si lo hacen, recurren al móvil. Para escuchar música utilizan Spotify u otras plataformas similares. Son así mismo seguidores asiduos de series de conocidas plataformas como HBO, NETFLIX, MOVISTAR +, etc.

En lo que se refiere a su competencia colaborativa y cooperativa, encontramos en todos ellos una buena disposición para realizar cada tarea que se les encarga. Ade- 
más, percibimos un buen espíritu cooperativo en el grupo, que igualmente ponemos de relieve, y nos ocupamos de fomentar desde el primer día de clase; dejando muy claro que será totalmente necesario, con el fin de llevar a buen puerto los objetivos deseados. Entendemos que la madurez que nuestros alumnos nos aportan, con sus respectivas experiencias laborales, de estudios y de intercambios estudiantiles internacionales previos y/o simultáneos a este estudio, facilitan el mismo, así como la interacción entre ellos y la docente.

\section{Fases de la metodología aplicada a la Propuesta}

Para abordar la Propuesta, se han seguido las etapas que se detallan a continuación.

Fase cero

Se divide en dos momentos:

\section{Indagación y preparación de material en materia de ciberseguridad básica}

a) Indagación y preparación de material actualizado en materia de ciberseguridad que nuestros alumnos puedan utilizar de manera cotidiana al encender sus dispositivos (ordenador, móvil, tablet...). Es decir, herramientas de privacidad y seguridad disponibles en la configuración de los mismos.

b) Indagación sobre herramientas de privacidad y seguridad que ofrecen los distintos navegadores: Mozilla, Edge, Linux, Chrome...

c) Estudio previo de las herramientas estatales españolas, francesas y del ámbito francófono centradas en la ciberseguridad y protección datos y de derechos de autores:

La Agencia Española de Protección de Datos (AEPD, 2021), la Lista Robinson (2019), INCIBE (Instituto Nacional de Ciberseguridad y su teléfono 017) (Díaz Moles, 2019), CEDRO, que vela por la protección de autores y editores (Corrales, 2021), el Teléfono de la esperanza (2021), la aplicación “Ana Bella" contra la violencia de género (Observatorioviolencia.org, 2018), y la Aplicación desarrollada por la web Maldita.es (Jiménez Cruz y Montes, 2017), que trabaja junto con la Fundación de Ayuda Contra la Drogadicción contra la desinformación y los bulos en la red, que lleva el nombre de la campaña "No more haters" (Jiménez Cruz y Montes, 2021), la Commission Nationale de L'Informatique et des Libertés (CNIL, 2021), la AFAPDF (Association Francophone des Autorités de Protection des Données Personnelles (CNIL, 2021), 
Comité Européen de la Protection des données (CEPD, 2021), (CNA) Conséil Supérieur de L'Audiovisuel.fr (Maistre, 2011), (Hadopi) Haute Autorité pour la Diffusion des Euvres et la Protection des Droits sur Internet (Rapone, 2021), Défenseur des droits.fr (Constance, 2011)

c.1)

- División entre webs desarrolladas en el sistema cultural español y aquellas elaboradas desde el entorno cultural francés y francófono.

- Elección entre ellas de las herramientas más adecuadas para ser utilizadas por nuestros estudiantes, futuros profesionales en un entorno francófono.

\section{Cuestionario inicial (consultar el Apéndice)}

2.1. Elaboración de un cuestionario inicial sobre el uso que nuestros estudiantes hacen de las herramientas TIC en relación con los Estudios Franceses, incluyendo las herramientas de protección de datos y webs colaborativas.

2.1.1. Realización del cuestionario inicial por el alumnado.

- Recopilación de Información sobre el nivel TIC del alumnado en relación con los Estudios Franceses.

- Recopilación de información sobre conocimientos básicos del alumnado en materia de ciberseguridad, herramientas de protección de datos y webs colaborativas.

\section{Fase 1}

3. Exposición del alumnado a herramientas básicas de ciberseguridad en su búsqueda cotidiana de documentación para los Estudios Franceses

Fase que a su vez se divide en seis:

3.1. Búsqueda, localización e identificación de herramientas de gestión de privacidad y seguridad en los propios dispositivos del aula TIC, móviles, tablets, etc.

3.2. Búsqueda, localización e identificación de herramientas de gestión de privacidad y seguridad en los navegadores web utilizados: Mozilla Firefox, Google Chrome, Microsoft Edge, etc.

3.3. Exposición del alumnado a webs oficiales, organizaciones sin ánimo de lucro, dedicadas a la protección del internauta y a velar por la integridad e igualdad de las personas, de autores y editores. 
3.3.1. Exposición del alumnado a webs oficiales, organizaciones sin ánimo de lucro, del entorno español dedicadas a la protección del internauta y a velar por la integridad e igualdad de las personas, de autores y editores, de modo que el alumnado comience a familiarizarse con este tipo de herramientas existentes en su propio entorno cultural conocido, dentro del sistema cultural español.

3.3.2. Exposición del alumnado a webs oficiales, organizaciones sin ánimo de lucro, dedicadas a la protección del internauta y a velar por la integridad e igualdad de las personas, dentro del ámbito francés y francófono. Una vez introducidos en el mundo de la ciberseguridad en la cultura española actual, se procede a aplicar dicha exposición del alumnado al mismo tipo de herramientas, en el entorno francés y francófono.

Una parte de nuestros alumnos van a realizar Erasmus en Francia durante el curso 2021-22, por lo que, entendemos que tanto el conocimiento previo de los mismos, antes de ir al país galo, como su posterior disposición a su consulta y manejo han de serles de gran utilidad; y podrían serles de gran ayuda en caso de un hipotético caso de acoso o ciberacoso o situaciones límite similares que pudieran poner en peligro su integridad física y o psicológica. Ciberacoso infligido sobre sus personas -en tanto que usuarios del espacio cibernético francés y francófono-, uso de datos por terceros...

3.4. Introducción al uso sistemático de mecanismos de protección de datos como práctica cotidiana en el aula antes de proceder a cualquier búsqueda web y/o uso de aplicaciones y herramientas TIC para los Estudios Franceses.

- Búsqueda, localización e identificación de herramientas de gestión de la privacidad y seguridad disponibles en las propias webs visitadas para la realización de trabajos académicos en Estudios Franceses. A modo de ejemplo introductorio, que ellos puedan luego aplicar, se practicará esta búsqueda, localización e identificación con las propias webs dedicadas a la ciberseguridad del internauta, con las que se ha trabajado en el punto 3.3 .

3.5. Empleo de la información detectada en el cuestionario inicial a la práctica docente en el uso de las TIC aplicadas a los Estudios Franceses.

- Análisis sistemático, por parte del alumnado, de los resultados obtenidos en materia de ciberseguridad en relación con los E.F. de cualquiera de las aplicaciones y/o entornos web visitados.

3.6. Comprobación de la incorporación hecha por nuestros alumnos a su acceso cotidiano a la red, a aplicaciones y herramientas adaptables al trabajo con Es- 
tudios Franceses del uso de herramientas de protección de datos y ciberseguridad.

a) Seguimiento continuado en cada una de las tareas del alumnado que incluyen búsquedas en Internet, sobre la consulta de herramientas de protección de datos previa a cualquier búsqueda. El alumnado tendrá que entregar, con cada tarea realizada para los Estudios Franceses, una ficha identificativa de los datos profesionales de cada web consultada.

b) Elaboración de un cuestionario final sobre el uso que nuestros estudiantes hacen de las herramientas TIC en relación con los Estudios Franceses, incluyendo las herramientas de protección de datos y webs colaborativas. (consultar el Apéndice)

Recopilación de Información sobre el nivel TIC del alumnado (a través de un cuestionario final).

c) Recopilación de información sobre conocimientos básicos del alumnado en materia de ciberseguridad (a través de dicho cuestionario final).

\subsection{Cuestiones planteadas en los cuestionarios inicial y final}

En el punto 2 de la Fase Cero de este estudio se entrega a los alumnos/as un cuestionario -que incluimos como Apéndice al final de este artículo- en el que se indaga sobre su posible conocimiento de herramientas TIC. Una vez realizados los cuestionarios iniciales de manera individual por los/las alumnos/as, se hace una puesta en común en el aula sobre los conocimientos generales del uso de las TIC del grupo universitario en su conjunto. De tal modo, se muestra a los propios alumnos cuáles son los puntos de partida de esta Propuesta de nivel cero. Trabajamos, en 2021, con alumnos de la Generación Z, nativos digitales, y ello nos lo corroboran las respuestas mostradas en los respectivos cuestionarios. Seguidamente, se indica a los/las alumnos/as que varias semanas antes de finalizar las clases, volveremos a entregarles el mismo cuestionario en blanco, de modo que ellos mismos puedan analizar la evolución de su conocimiento de las herramientas TIC, probablemente aplicables a los Estudios Franceses, de las lenguas y culturas en general. A este respecto, en el mencionado cuestionario que incluimos como Apéndice al final de este artículo, se sondea su uso de webs/apps relacionadas con el aprendizaje de las lenguas extranjeras y cuáles entienden que podrían ser aplicables a los Estudios Franceses. Se les inquiere, igualmente, en dicho cuestionario, sobre su posible uso de redes sociales, su conocimiento de youtubers, influencers, streamers. Se les cuestiona sobre las posibles ventajas e inconvenientes de Internet. Se les pregunta su opinión sobre el ciber acoso. Además, como la base de nuestro trabajo indica, se les consulta acerca de su conocimiento de organismos relacionados con la protección de datos. En el mismo sentido, se les interpela sobre sus 
hábitos a la hora de consultar y utilizar cualquier sitio web: si tienen costumbre de leer las condiciones de uso de apps, redes sociales, webs, blogs, antes de aceptar las cookies o cualquier otro sistema de rastreo de datos e identidad, antes de instalarse una app, descargarse o escuchar una canción, un vídeo, una película, un programa. Al hilo de lo anterior, se recaba su opinión sobre cuestiones como la publicidad en relación con las búsquedas realizadas en Internet, y los datos sobre preferencias personales que las webs recogen al hacer cualquier búsqueda en la red. Se les cuestiona si creen que las visitas a las páginas, el uso de las redes, de las descargas, etc., es gratis. Así mismo, con objeto de llevar este estudio más allá de una concienciación del alumnado del uso cotidiano que hacen de la red, de la exposición de sus datos personales y de cualquier tipo, de la no gratuidad del uso de la red, como elementos de utilidad añadidos a las TIC aplicables al aprendizaje de las lenguas y culturas, en el mismo cuestionario incluimos un apartado dedicado al conocimiento de las webs colaborativas.

\subsection{Conclusiones de las respuestas del alumnado al cuestionario inicial que propician el paso a las siguientes fases del estudio}

Dicho cuestionario inicial nos ha permitido detectar los niveles de conocimiento de nuestros alumnos sobre la materia: Las TIC aplicadas a los Estudios Franceses. Un conocimiento que entendíamos al tratarse de personas pertenecientes a la Generación Z, podría no ser precisamente precario. El cuestionario inicial confirma nuestra intuición formativa y pedagógica de base.

En los cuestionarios inicial y final, que han servido de base a esta propuesta experimental, como temas básicos se han tratado la relación de las TIC con los Estudios Franceses, los conocimientos previos y posteriores del alumnado en relación con las TIC, la ciberseguridad, el acoso, el ciberacoso, la cooperación, la igualdad y no violencia entre los seres humanos.

De tal modo, los investigadores tendrán acceso a esas respuestas, y podrán comprender el alcance del estudio desde su planteamiento previo; pasando por las etapas de aplicación y desarrollo, para llegar a las conclusiones aportadas con las respuestas de los cuestionarios finales.

Observando en julio de 2021, con mayor perspectiva temporal las preguntas planteadas en los cuestionarios inicial y final, encontramos que sería interesante introducir una mayor concreción en algunas de estas preguntas; que no diesen pie a una respuesta con monosílabos (sí/no), sino a respuestas más extensas. Hacemos esta observación con la perspectiva que da el análisis de resultados de la Propuesta, gracias tanto al cuestionario final como al seguimiento continuado de la misma a través de todo un cuatrimestre. Apreciamos estos aspectos, teniendo en cuenta que el interés final de los cuestionarios utilizados como 
Anales de Filología Francesa, n. ${ }^{\circ}$ 29, 2021

ESMERALDA VICENTE CASTAÑARES

herramienta indagatoria es conocer el manejo de TIC y herramientas de ciberseguridad de nuestro alumnado en relación con los E.F.

\section{Objetivos obtenidos a corto plazo con nuestra Propuesta}

En estos escasos meses del segundo cuatrimestre de 2021 hemos expuesto al alumnado participante en el estudio, de manera sistemática, a consultar y hacer uso de los escasos elementos y medios de ayuda existentes, hoy día, en materia de protección de datos a nivel usuario. Estas mismas herramientas redundan en evitar el acoso (no sólo de compañeros, conocidos, desconocidos, sino también de empresas, publicidad...) al que, los alumnos, como simples usuarios de la red podrían verse expuestos.

¿Qué elementos pedagógicos y competencias han adquirido y/o desarrollado nuestros alumnos con esta Propuesta de nivel cero en la didáctica de los Estudios Franceses desde las TIC? ¿Qué objetivos hemos conseguido a corto plazo?

En primer lugar, un acrecentamiento de su disposición propia, sin necesidad de guía de la docente, con cierta intriga y diligencia para la realización de tareas de prevención en ciberseguridad básica. Han manejado, entre otras, algunas que consideramos esenciales, tales como consultar políticas de privacidad y cookies, ajustar sus parámetros de búsqueda en móviles y ordenadores, configurar los elementos de privacidad y seguridad que ofrecen navegadores y webs, etc. En definitiva, detectar quién está tras el contenido ofrecido en las webs (personal de atención a usuarios, dirección postal y electrónica, teléfono, CIF).

En segundo lugar, entendemos que ya son capaces de ejercer de manera autónoma un registro de los datos de las webs consultadas. Han pasado de ser meros "productos pasivos" del uso de la red y los componentes tecnológicos que en ella se ofrecen, del uso de sus móviles y ordenadores, a "sujetos activos". Lo corrobora su evidente inclinación a utilizar esas herramientas con las que, aunque sea mínimamente, pueden tratar de gestionar su privacidad. Queremos recalcar este "mínimamente", ya que, nosotros mismos, antes de presentarles a ellos cada una de estas herramientas, de las que disponemos en móviles, ordenadores, y en la red, que deberían realmente poder garantizarnos los niveles de privacidad deseados, no lo hacen sino en cierta medida. Por ejemplo, la Lista Robinson, te permite elegir no recibir publicidad o en el correo, o por llamadas telefónicas, pero no por ambos canales comunicativos. De igual modo, en lo referente a la configuración de la privacidad en ordenadores, tabletas, móviles, etc., hemos detectado que los mecanismos puestos a disposición de los usuarios son muy limitados, y así lo hemos puesto en conocimiento de nuestro alumnado. Por consiguiente, ellos mismos, a la hora de intentar configurar estos parámetros, criterios de búsqueda, preferencias, u otras opciones, en numerosos casos meramente simbólicos, han podido comprobar que, de una manera u otra, 
el sistema tecnológico está diseñado, y cada vez más se encamina a captar datos y más datos, quieran los usuarios o no. En efecto, para acceder a los contenidos, los alumnos han podido comprobar que, al intentar configurar preferencias, cookies, etc., en buena parte de los casos, la web no deja acceder a todo o parte del contenido si se rechazan todos los parámetros. Con lo cual, un usuario de elementos tecnológicos de nivel básico (es decir, que no sea un programador) está cada vez más expuesto en su intimidad personal y profesional.

En todo caso, hemos de recalcar que la exposición permanente a webs relativas a la protección de datos, así como la utilización continuada del resto de herramientas encaminadas en la misma dirección, han servido para concienciarles acerca de los peligros del uso de la red y de su propia capacidad personal para detectarlos. Lo que es más importante, ya no sólo se percatan de la intromisión que cualquier web, red social, pudieran hacer de su intimidad en el uso del ordenador en el aula. De tal modo, han pasado a incorporar el desarrollo de esta capacidad detectora, a su cotidianidad en la utilización de aplicaciones en su móvil. Competencia que, probablemente, intuitivamente ya tendrían, y que nosotros únicamente hemos tratado de contribuir a poner de manifiesto, entre otras cuestiones, sobre el ciberacoso: traducido no sólo a ámbitos relacionales, sino también al que nos somete la red como usuarios con la publicidad empresarial y la captura de datos personales, valiéndose ésta de la necesidad, del deseo -y la mayor parte de las veces- de la prisa con la que nuestro alumnado quiere acceder a los contenidos ofrecidos en la web.

Finalmente, al hilo de la velocidad de acceso a las webs, quisiéramos destacar el desarrollo de su competencia en materia de protección de datos personales. Entendemos que la observación continuada que les hemos propuesto, en lo relativo a la práctica de la velocidad de acceso a las webs como trampa que implica captación masiva de datos personales, ha dado sus frutos. Una cuestión que, a nuestro entender, han aprendido, ya que son bastante más conscientes de lo que ellos mismos pueden vivenciar, con el uso de los móviles, del hecho de verse "invadidos" por anuncios de un producto que consultaron días, semanas u horas atrás.

\section{El uso crítico de las TIC en relación con nuestra Propuesta}

Nos congratula el hecho de haber destacado en nuestra Propuesta, como punto de partida, la necesidad de los docentes de considerar el aprendizaje informal, autodidacta de los estudiantes nativos digitales en su justa medida. Coincidimos en esta premisa con Ana Viñals Blanco y Jaime Cuenca Amigo, que en su artículo El rol del docente en la era digital entienden éste como "un tipo de aprendizaje que [...] se reconoce más bien poco desde el ámbito formal” (Viñals Blanco y Cuenca Amigo, 2016: 105). La Propuesta de nivel cero en la didáctica de los Estudios Franceses desde las TIC que aquí presentamos 
es, así mismo, fruto de una combinación de aprendizaje formal e informal y autodidacta nuestro.

Viñals Blanco y Cuenca Amigo destacan igualmente la necesidad de tener en cuenta en el rol de los docentes en la era digital el "conectivismo", que:

Se fundamenta [...] en la creación de conexiones. Según [George Siemens, 2006] el conectivismo es la integración de principios explorados por las teorías del caos, las redes, la complejidad y la autoorganización. (Viñals Blanco y Cuenca Amigo, 2016: 105-106)

Precisamente, coinciden con nosotros en detectar el exceso de contenidos accesibles en Internet "de ahí la necesidad de construir una red de aprendizaje (Entornos Personales de Aprendizaje-PLE), de los cuales podamos entrar y salir en cualquier momento" (Viñals Blanco y Cuenca Amigo, 2016: 105-106). Al reconocimiento de la competencia digital autodidacta del alumnado en la era digital, del conectivismo, y de los Entornos Personales de Aprendizaje, añaden el ser multitarea en que ha de convertirse el docente:

Entrenador, [...] guía [...] y experto en instrucción [...]

Los docentes, además de tener que adaptar las metodologías de enseñanza al nuevo entorno, tienen ante sí el reto de adquirir conocimientos, habilidades y actitudes digitales que motiven al alumnado a hacer un uso crítico de la tecnología no solo en el aula, sino también en casa, en su vida social y en sus entornos de ocio. (Viñals Blanco y Cuenca Amigo, 2016: 105-106)

A este respecto del uso crítico de la tecnología, que viene a ser la base de nuestra Propuesta de nivel cero que aquí exponemos, traemos igualmente a colación el saber de Adela Cortina, Catedrática de Ética de la Universidad de Valencia y Doctora Honoris Causa por la Universidad de Murcia. Para Cortina, "el progreso tecnocientífico tiene que ir de la mano del progreso ético". En la Revista Campus Digital de la Universidad de Murcia, añade la Académica de ciencias morales y políticas que la ética "sirve para formar ciudadanos, para formar personas que busquen la justicia y la felicidad, y para formar ciudadanos con sentido de la dignidad humana." (Vera Nicolás, 2016). Justamente, nosotros entendemos como parte de nuestra labor docente, la necesidad de agregar a los conocimientos técnicos de nuestro alumnado, la parte de ética que vemos tan necesaria en el uso de las TIC. Conceptos como resiliencia, aprendizaje cooperativo, empatía, diversidad, libertad, igualdad, creemos que podrían no estar tan desarrollados como quisiéramos en tanto que docentes; tanto en el imaginario como en la cotidianidad de nuestros alumnos. El cuestionario presentado en punto 4 de este artículo (4. Fases de la metodología aplicada a la Propuesta), como herramienta base, nos servirá también para incorporar estos elementos y valores básicos al "planeta Z" en el que interaccionan nuestros alumnos. Por ello, la introducción en el cuestionario de un 
concepto como el acoso virtual nos parecía del todo pertinente, e íntimamente relacionado con las herramientas que queríamos proponer al alumnado, como base experimental en la que sumergirse a la hora de abordar el uso consciente, responsable y lo más seguro posible de las TIC.

Nosotros queríamos introducir en la rutina diaria de uso de las TIC de nuestro alumnado, y concretamente de todas aquellas que requieren la utilización de Internet, la consulta sistemática de elementos de gestión de datos como: la política de privacidad y cookies, propiedades, criterios de búsqueda, configuración, privacidad y seguridad de webs y apps que visiten. Una rutina en la que ellos mismos desarrollen un interés por buscar, detectar, encontrar quién/es está/n tras la página web o app que consultan: dirección postal, correo electrónico y teléfono, CIF (si se proporciona). Además, partimos de la base de que nuestros alumnos no son desarrolladores de software, aunque sí están muy motivados e interesados en descubrir estos parámetros referidos a su privacidad y a la de los demás. Más aún, estamos introduciéndoles en el uso de estos mecanismos de protección de datos con el afán de que se acostumbren a usarlos, no solo en el presente, en nuestra presencia en clase, o durante este proyecto de inmersión en el otro lado de la cara visible de las webs visitadas. Ahora bien, ellos, por su deseo de "devorar" contenidos nuevos, apps, redes, blogs, canales de youtube, influencers nuevos, y todo lo que la red les ofrezca "gratis", se sumergen sin pudor y ofreciéndose como "producto". Empero, el cuestionario inicial ya mencionado nos ha permitido, así mismo, constatar que conocen la teoría de todas estas cuestiones referidas a la ciberseguridad en relación con evitar el acoso. Incluso, hemos corroborado este punto al volverles a plantear la cuestión del ciberacoso en el cuestionario medio-final que les hemos hecho. Sus comentarios han ratificado sus opiniones iniciales.

\section{EI uso ético de las TIC y su impacto planetario. La relevancia de nuestra Propuesta}

A este respecto de la captación de datos, hemos puesto igualmente de manifiesto en esta Propuesta la cuestión del necesario almacenaje de las cantidades ingentes de datos que genera la red. Almacenaje que necesita de unas infraestructuras cada vez más sofisticadas, gigantescas y unidas a la salud medioambiental y climática del planeta; como ha dejado patente el CEO de Tesla, Elon Musk, al anunciar que se desmarca del uso del bitcoin por la ingente huella de carbono que ya está dejando en la Tierra (El confidencial.es, 2021). Lo estamos viendo incluso en nuestro país, con la planificación de la descentralización de las infraestructuras de almacenamiento de datos que ya se está produciendo. Como paradigma tenemos el traslado del centro de procesos de datos de la Seguridad Social a Soria (Rentero, 2021). El calentamiento del clima nos está saliendo caro, así es que hay que abaratar costes en electricidad, usando la refrigeración natural que aún proporcionan las zonas frías del planeta para 
poder mantener estos gigantescos equipos en funcionamiento. Somos dependientes de ellos para cualquier gestión cotidiana. En La salud está ligada al planeta, artículo publicado en El médico interactivo.com, Nekane Lauzirika, recoge las reflexiones y acciones de algunos integrantes de la recién creada plataforma Sanidad \#PorElClima, que impulsa la acción climática en el sector sanitario:

\begin{abstract}
Por medio de un formulario, según la organización, la plataforma ayuda a cada usuario a identificar las medidas que ya ha implementado en diferentes áreas de impacto, como son agua, energía, consumo, movilidad, huella de carbono y gestión de residuos.

A partir de aquí, obtiene un informe personalizado que detalla su grado de sostenibilidad climática, lo que permite trazar una hoja de ruta con sugerencias para posibilitar la descarbonización. (Lauzirika, 2021)
\end{abstract}

Por ello, entendemos que unos seres humanos como nuestros alumnos, pertenecientes a la Generación Z, que más que nacer entre árboles, lo han hecho entre tablets, wiis, consolas, teléfonos "inteligentes", necesitan de nuestra labor docente para traerles de nuevo al planeta Tierra, en el que puedan encontrar sus verdaderas raíces. Todavía tenemos tiempo para conseguirlo, aunque no demasiado, y no podemos desperdiciarlo. Las paradojas de lo que ocurre en nuestra sociedad lo ponen de manifiesto cada día. En abril de 2021, mientras faltaba oxígeno para millones de seres humanos en la India, el Perseverance estaba fabricando oxígeno en Marte. Y no era gracias a la tecnología, o al menos no única y exclusivamente. Más bien y, sobre todo, el Perseverance ha llegado a fabricar oxígeno en Marte gracias a las mentes humanas inteligentes que han conseguido fabricarlo. Ya en 2018, Cortina, en Ciudadanía digital y dignidad humana parecía anticiparse a posibles eventos de envergadura colosal, abordables gracias a las alianzas entre cerebros humanos y nuevas tecnologías en el mundo laboral, como lo ha sido este gran hito humano del "Amartizaje”. Eso sí, nos prevenía ante el peligro de quedarnos estancados en un posible estado contemplativo y fantasioso, al respecto de las "Inteligencias" Artificiales y sus innumerables capacidades, para dejar a un lado el hecho de que también podrían ser peligrosamente programadas con sesgos discriminatorios creados por humanos:

Es imposible predecir el curso que van a seguir los avances tecnológicos, pero sí que podemos anticipar para qué mundo los queremos [...]. La razón moral debe ir por delante de la razón técnica. [...] El objetivo es construir una ciudadanía digital de pleno derecho, lo cual exige hacer frente a retos como la ciberseguridad, la protección de datos personales, la privacidad de los usuarios, la accesibilidad, la propiedad y la gestión de los datos o la mejora de las capacidades digitales. Pero también abordar cuestiones tan complejas como quién será responsable de un fallo de competencia robótica, cómo enfrentar el hecho de que las máquinas también tienen sesgos en sus decisiones o el problema de que los algoritmos carezcan de contexto. (Cortina, 2018) 
Por ello, con nuestra Propuesta, consistente en abordar sistemáticamente cualquier contenido web desde el conocimiento de quién está tras esa web, en utilizar las herramientas disponibles a los usuarios para su propia protección de datos, antes de acceder a dichos contenidos, nosotros queríamos desmontar un mito a ojos de nuestros alumnos. Ese mito no es otro que el de considerar inteligentes a teléfonos y demás dispositivos "inteligentes", así como a la, entendemos probablemente sobrevalorada "Inteligencia" Artificial. De tal modo, con nuestra Propuesta, queríamos poner de manifiesto que son los cerebros humanos de los seres humanos los verdaderos protagonistas del desarrollo tecnológico. A su vez, queríamos insistir en que ese desarrollo ha de ir ligado a una consciencia del respeto a la especie humana en su conjunto, y al planeta en el que ésta vive, porque, de momento, no tenemos otro. Con este deseo nos unimos así a las enseñanzas éticas transmitidas por Adela Cortina y Eva González de Sanidad por el clima, respectivamente. Los posibles sesgos, en alianza estrecha con la matemática algorítmica, podrían poner en peligro la natural interacción humana. De la misma manera, enfocar a los seres humanos hacia encierros o sistemas de pensamiento unificado, recortar sus derechos, y otras cuestiones morales y éticas, podrían darse como consecuencia de "descargar" a los humanos desarrolladores de sesgos algorítmicos de su responsabilidad ética, para trasladársela a las máquinas. Cortina, en Ética de la inteligencia artificial desde Europa, avisa de la responsabilidad de la especie humana de su propio autocuidado moral y ético, en un mundo hiperconectado:

\begin{abstract}
¿Qué significa "ética de la inteligencia artificial”? ¿Es la que deben practicar desde sus valores los sistemas inteligentes, es decir, las máquinas, los algoritmos, los robots, o es la ética que los seres humanos deberíamos adoptar para servirnos de los sistemas inteligentes? [...] el elemento directivo es la persona humana que se sirve de los sistemas inteligentes para tratar gran cantidad de datos, incluso para aprender de sus "experiencias". Es en este tipo de inteligencia artificial en el que actualmente nos encontramos. No se trata, pues, de una ética de los sistemas inteligentes, sino de cómo orientar de forma ética el uso humano de estos sistemas para resolver problemas. En este quehacer la UE tiene que ser pionera, uniendo al progreso tecnocientífico un liderazgo ético para que sea posible pasar de las excelentes declaraciones con que cuenta a las efectivas realizaciones. (Cortina, 2019)
\end{abstract}

Conseguir que nuestros alumnos sean conscientes, al reconocer la existencia del acoso y el ciberacoso, que no han de practicarlo, que no han de ser meros espectadores de aquél que pudieran sufrir otros seres humanos; así como del acoso al que están expuestos con la captura de sus datos en la red, nos lleva a otro de los conceptos fundamentales de los valores que queríamos transmitirles y de los que destaca la propia Cortina: la libertad y la igualdad. Pues el exceso de libertad de unos, podría coartar la libertad y la igualdad de otros. Mientras que todo ello, en medidas proporcionales, podría contribuir a establecer una sinergia que ayude a los seres humanos a interactuar sin denigrar a los otros. Así pues, 
entendemos muy necesaria nuestra labor como docentes en todo el proceso de concienciación relativo al conjunto de conceptos que acabamos de mencionar, y que conforman un todo que se resumen en: conseguir como objetivo que la Generación $Z$ entienda y reflexione sobre la necesidad de establecer un equilibrio entre evolución tecnológica y evolución humana y de la naturaleza. ¿Por qué y para qué? Para que la generación que ya les está sucediendo entienda también esta necesidad. Ello podría traducirse en saber encontrar el equilibrio entre velocidad tecnológica y ritmo natural y humano planetario; de modo que siga habiendo seres humanos que se respeten entre sí, que sean capaces de respetar la privacidad de los otros, y de reclamar la suya. Capaces no sólo de compartir cada microsegundo de su existencia experiencias tan increíblemente "geniales" como peligrosamente privadas en Youtube, Instagram, Tik Tok, ... sino también de compartir el suelo en el que pisan cada día, el aire que respiran, las miradas presenciales que pueden intercambiar, si levantan la vista de las pantallas en línea, miradas de todo tipo: alegres, como el postureo que también y sobre todo forma parte de la cara amable de las redes, pero igualmente miradas tristes, preocupadas, angustiadas, desesperadas incluso. De modo que sean así competentes para identificar la necesidad de otros seres humanos, y alejarse, ciertamente, del yoísmo vendido por cualquier espacio de la red de redes, del que confiamos hayan dejado de ser, o van quizá a serlo, paulatinamente, meros "productos pasivos", para estar dispuestos a convertirse en "sujetos activos". Personas aptas para transmitir a otros seres del planeta con los que interaccionan, así como a la generación que les sucederá, ese afán de "actividad" y de "intercambio de valores" como los que se entrenan, por ejemplo, desde la Fundación Ana Bella.org para mujeres maltratadas, ya presente en colegios de Primaria, que, a su vez ha desarrollado la Aplicación Ana Bella, para apoyar a mujeres en su proceso de denuncia (Observatorioviolencia.org, 2018).

En definitiva, quisiéramos que nuestros alumnos fuesen capaces de levantar la vista de las pantallas y empatizar, ponerse en la piel de los otros, no únicamente con el espíritu consumista de los algoritmos creados para captación de datos para la publicidad en la red por las "Inteligencias" Artificiales. Sino haciendo uso de la tecnología humana de la que disponen en su propio cerebro, mostrando que son tan importantes y necesarios como la IA, porque son, simple y llanamente, los imprescindibles RRHH que tan olvidados están últimamente por la ciencia del planeta en el vivimos, la Tierra. Recursos Humanos que son prejubilados en masa por grandes empresas que cotizan en el IBEX'35, porque a sus directivos no les interesa que miren a los ojos a los clientes, ya que resulta más fácil y económico captarles a golpe de acoso virtual y telefónico.

Todo esto nos lleva a preguntarnos ¿Por qué tanta insistencia por parte de los órganos institucionales en el uso de las TIC en la enseñanza en general, y en la enseñanza universitaria en particular? ¿Y si tras una supuesta y obligatoria necesidad de innovación educativa ligada a las TIC no pudiera haber ciertos intereses de lobbies empresariales y económicos 
globales? ¿Llenar los centros educativos de webs de rápido acceso a contenidos ligados indefectiblemente a publicidad, no sería un método efectivo para una captación masiva de clientes? A propósito de esta cuestión, hemos consultado dos investigaciones. Una de ellas, en relación con un estudio de casos sobre la implantación de uso de TIC con toda su dotación de ordenadores en Institutos de Enseñanza Secundaria de Canarias. La otra trata una implantación similar en Grados de toda índole en universidades francesas. Ambas vienen a apuntar que, hoy día, ya no hay opción para la docencia y el aprendizaje en las aulas sin pasar por el uso de las TIC. En las conclusiones sobre el proyecto MEDUSA del gobierno de Canarias, Área Moreira, comenta que:

Se trata de un proceso de la innovación a gran escala que sigue un modelo de centro a periferia en el sentido de que la necesidad de la innovación es provocada externamente desde instancias administrativas. De este modo el profesorado juega un papel de ejecutor de las decisiones adoptadas en un nivel superior por el [...] equipo de expertos que diseñan y difunden el proyecto (administración central) [...] En este sentido, los hallazgos encontrados para los casos estudiados son similares a lo apuntado por los estudios nacionales e internacionales [...] existe una notoria presencia de la tecnología, pero ésta por sí misma no genera procesos sustantivos de cambio metodológico en las prácticas de enseñanza y aprendizaje. (Area Moreira, 2010: 94-95)

Así mismo, Jérôme Valluy (2012) analiza el estudio TIC et métiers de l'enseignement supérieur - Emergences, transformations (2011) que, a su juicio, ha hecho un análisis sesgado del uso de las TIC en universidades francesas; encuestando únicamente al profesorado y no a la administración de la universidad ni a los alumnos. Eso sí, Valluy redunda en señalar que:

L'émergence de l'Internet et des applications informatiques utilisables dans l'enseignement supérieur et la recherche offrent certes de magnifiques opportunités dans le travail universitaire, mais cela ne veut pas dire que les besoins réels soient les mêmes dans toutes les disciplines, tant du point de vue du choix des outils que de l'intensité de leurs usages [...] la position dominante des enseignants-chercheurs sur la gouvernance des universités [...] est précaire, constamment menacée par de nouvelles dépendances: cela est flagrant sur le plan financier où l'emprise des technocraties comptables sur les choix scientifiques et pédagogiques, à travers la gestion et le contrôle budgétaire, est considérable. Celle des technocraties informatiques s'annonce toute aussi importante sur les choix scientifiques (portails, bases de données, statistiques...) et pédagogiques (cadrages techniques des communications professeurs/ étudiants). (Valluy, 2012)

\section{Conclusiones}

Según muestra El Periódico.com en un artículo de junio de 2014 titulado "Acoso telefónico". "Entra en vigor la ley que limita el horario de las llamadas comerciales", ya desde 2014 las empresas tenían limitado, por ley, el "acoso telefónico" (El Periódi- 
co.com, 2014) a los ciudadanos. Sin embargo, una parte de éstas podrían haber estado incumpliendo parte de esos límites, que ahora en julio de 2021, se vuelven a poner de manifiesto y a intentar acotar desde el Ministerio de Consumo español. Precisamente, La Lista Robinson, una de las herramientas que hemos utilizado en nuestra propuesta, se presenta actualmente en los medios como un ejemplo de freno al acoso publicitario. En todo caso, desde la perspectiva de los usuarios universitarios, hemos de señalar que la relevancia de nuestra Propuesta a nivel cero queda patente al observar que la ciberseguridad se incluye en el currículo del nivel de certificación básico (A2) del Marco de referencia de la Competencia Digital Docente (BOE n 191, 2020). Ello viene a corroborar la necesidad de los usuarios universitarios de contar con competencias básicas de ciberseguridad antes de manejar cualquier herramienta y contenido TIC. O sea, en su nivel cero como usuarios TIC.

Así pues, tal y como nos lo hemos planteado en este estudio desde el principio, si debemos dar por hecho que hemos de convivir en nuestras aulas universitarias, sí o sí con las TIC, tratemos de hacerlo con conciencia crítica, ética y valores. Podemos recordar cada día que la pedagogía, probablemente, ya existía en aquellos bisontes pintados en Altamira; la ética ya estaba acaso de algún modo presente en la construcción de entornos monumentales como el Machu Pichu, Stonehenge, los castros y otros variopintos patrimonios olvidados cercanos a nosotros; de nuestro diminuto planeta, que durante siglos dejaron menos huella de carbono de la que nosotros vamos a dejar quizá, en escasas décadas, simplemente con el almacenamiento de datos en la nube. Hemos de reconocer que hemos encontrado ciertas limitaciones tecnológicas al tratar de introducir a nuestro alumnado en el intrincado mundo de la protección de datos en el uso de las TIC para los Estudios Franceses. Restricciones que hemos identificado como estudiadas desde la configuración de dispositivos, navegadores, webs, por las propias marcas desarrolladoras de las herramientas básicas de gestión de la privacidad que las ofrecen. Nuestro alumnado ha podido comprobar, de primera mano, dichos condicionamientos, al intentar hacer uso de las herramientas de autogestión de la privacidad que les hemos presentado. Pese a todo ello, entendemos que esta Propuesta de nivel cero en la didáctica de los Estudios Franceses desde las TIC, ha sido productiva y podría contribuir a la protección de los internautas universitarios desde su primer contacto con las TIC para los Estudios Franceses.

\section{Referencias bibliográficas}

AEPD. 2021. "Agencia Española de Protección de Datos". Madrid, AEPD: <https://www. aepd.es/> [ 02/01/2021].

Área Moreira, Manuel. 2010. "El proceso de integración y uso pedagógico de las TIC en los centros educativos. Un estudio de casos” en Revista de Educación, nº 352, 77-97. 
ArILlo Crespo, Magdalena.1992. "Implicaciones didácticas del Diseño Curricular Base en el Área de Lenguas Extranjeras", en González Nieto, Luis y Magdalena Arillo Crespo. Implicaciones didácticas del DCB en "Lengua Castellana y Literatura" y "Lenguas Extranjeras". Zaragoza, Universidad de Zaragoza, ICE, Educación Abierta, 27-70.

BARBot Marie-José y Luc MAssou (Dir.). 2011. TIC et métiers de l'enseignement supérieur - Emergences, transformations. Nancy, Presses Universitaires de Nancy in Questions de communication n¹4, Série "Actes".

BOE. 2020. III. Otras Disposiciones. Ministerio de Educación y Formación ProfeSIONAl. Anexo: Acuerdo de 14 de mayo de 2020, de la Conferencia de Educación sobre el marco de referencia de la competencia digital docente.

BOE no 191, de 13 de julio de 2020. Sec. III, 50638.

CEPD. 2021. “Comité Européen de la Protection des données”. Bruselas, CEPD: <https:// edpb.europa.eu/edpb_fr/> [12/01/2021].

CNIL. 2021. "Commission nationale de l'informatique et des libertés". Paris, CNIL: $<$ https:// www.cnil.fr/> [08/01/2021].

Corrales, Jorge (dir.). 2021. "CEDRO.org. Funciones. Defensa". Madrid, Cedro.org: $<$ https://www.cedro.org/cedro/funciones/defensa $>$ [ 15/01/2021].

Cortina, Adela. 2018. "Ciudadanía digital y dignidad humana" en El País.es, Tribuna, [28/03/2018-00:00 CEST]: <https:/elpais.com/elpais/2018/03/22/opinion/1521737007_854105.html> [23/01/2021].

CortinA, Adela. 2019. "Ética de la inteligencia artificial desde Europa" en El País. es, Tribuna, [06/06/2019 - 00:00 CEST]: <https://elpais.com/elpais/2019/06/05/opinion/1559729489_306891.html> [23/01/2021].

Díaz Moles, Rosa (Dir.). 2019. “¿Qué es INCIBE?”. León: S.M.E. Instituto Nacional de Ciberseguridad de España M.P., S.A.: <https://www.incibe.es/que-es-incibe> [02/01/2021].

Denis, Marie-Laure (dir.) 2021. "Commission nationale de l'informatique et des libertés", CNIL. Paris: CNIL: <https://www.cnil.fr/> [08/01/2021].

Denis, Marie-Laure (Dir.) 2021. “Association Francophone des Autorités de Protection des Données Personnelles, AFAPDP”. Paris, CNIL: <https://www.cnil.fr/fr/definition/afapdp/> [09/01/2021].

El CONFIDENCIAL.es. 2021. "Elon Musk hunde el bitcoin al anunciar que Tesla deja de aceptarlo como pago" en El Confidencial.es, [13/05/2021 - 10:31 Actualizado: 13/05/2021 13:32]: <https://www.elconfidencial.com/mercados/2021-05-13/elon-musk-hunde-la-cotizacion-del-bitcoin-al-anunciar-que-tesla-deja-de-aceptarlo-como-forma-de-pago_3078432/> [13/05/2021].

EL PERIÓDICO.com. 2014. “Acoso telefónico". "Entra en vigor la ley que limita el horario de las llamadas comerciales" in El periódico.com, Sociedad, [13 /06/2014. Actualizada 15 /06/ 2014 - 03:20]. https:/www.elperiodico.com/es/sociedad/20140613/ entra-en-vigor-la-ley-que-limita-el-horario-de-las-llamadas-comerciales-3302075> [10/01/2021]. 
González, Manuel, Ernest Marco y Toret Medina. 2020. "Informe de iniciativas y herramientas de evaluación online universitaria en el contexto del COVID-19". Madrid. Ministerio de Universidades, Gabinete del ministro.

Jiménez Cruz, Clara y Julio Montes (cofund.) 2017. "Maldita.es”. Madrid: Maldita.es: $<$ https://maldita.es/> [24/01/2021].

Jiménez Cruz, Clara y Julio Montes (cofund.) 2021. "FAD y Maldita.es lanzan la campaña de sensibilización social para que adolescentes y jóvenes aprendan a identificar los discursos de odio. No more haters. ¡Rompe la cadena del odio!” en Maldita.es, Nosotros. Madrid: Maldita.es.: <https://maldita.es/nosotros/20210513/fad-y-malditaes-lanzan-la-campana-desensibilizacion-social-para-que-adolescentes-y-jovenes-aprendan-a-identificar-los-discursos-de-odio/> [24/01/2021].

Lista Robinson. 2019. "Lista Robinson.es". Asociación Española de la Economía Digital. Barcelona: Adigital: <https://www.listarobinson.es/> [03/01/2021].

LAUZIRIKA, Nekane. 2021."La salud está ligada al planeta" in El médico interactivo.com. [21/04/2021. 3:58 pm. Madrid]: <https://elmedicointeractivo.com/la-salud-esta-ligada-alplaneta/> [30/04/2021].

Maistre, Roch-Olivier (dir.). 2011. “(CNA) Conséil Supérieur de L’Audiovisuel.fr”. Paris, Conséil Supérieur de L’Audiovisuel: <http://www.csa.fr> [26/01/2021].

OBSERVATORIOviolencia.org. 2018. "Buena Práctica contra la violencia de género: App Ana Bella". Fundación Mujeres Actualidad, DESTACADAS, Sociedad Civil, violencia de genero 0. [07/12/2018]: <https://observatorioviolencia.org/buena-practica-contra-la-violencia-degenero-app-ana-bella/> [13/01/2021].

Rapone, M. Denis. (Dir.). “(Hadopi) Haute Autorité pour la Diffusion des Euvres et la Protection des Droits sur Internet”. Paris, Hadopi: <https:/www.hadopi.fr/> [20/01/2021].

Rattray, Ben y Mark Dimas. 2021. "Política de privacidad". Barcelona, Change.org, Servicios Promocionales España, S.L.: $<$ https://www.change.org/policies/privacy $>$ [16/01/2021].

Rentero, Antonio. 2021. "La Seguridad Social traslada su centro de proceso de datos a Soria para ahorrar en refrigeración" en Silocon.es, Actualidad TI, Noticias Cloud [26 /02/ 2021, 19:34h]: <https://www.silicon.es/la-seguridad-social-traslada-su-centro-de-procesode-datos-a-soria-para-ahorrar-en-refrigeracion-2433911> [27/02/2021].

Rivière, Constance (Dir.). 2011. "Défenseur des droits.fr". Paris, Défenseur des droits: $<$ https://www.defenseurdesdroits.fr $>$ [20/01/2021].

Siemens, G. 2006. Knowing Knowledge: <https://amysmooc.files.wordpress.com/2013/01/ knowingknowledge_lowres-1.pdf $>$ [10/01/2021].

Teléfono De la Esperanza. 2021. “Teléfono de la esperanza”. Madrid, Teléfono de la esperanza: <https://telefonodelaesperanza.org/> [09/01/2021].

VAlLuY, J. 2012. "Vers une pédagogie numérique à l'université? Compte-rendu et discussion de l'ouvrage TIC et métiers de l'enseignement supérieur et de la recherche - Émergences, transformations (nov. 2011)", Recueil Alexandries, Collections Recensions: <http://www.reseau- terra.eu/article1234.html $>$ [10/01/2021]. 
Anales de Filología Francesa, n. ${ }^{\circ}$ 29, 2021

UNA PROPUESTA DE NIVEL CERO EN LA DIDÁCTICA DE LOS ESTUDIOS FRANCESES DESDE LAS TIC

Vera NiCOLÁs, Pascual. 2016. "El progreso moral no ha estado al mismo nivel que el progreso técnico (Adela Cortina, nueva doctora honoris causa por la Universidad de Murcia)" en Revista Campus Digital, Universidad de Murcia, Entrevistas y Reportajes: <https:// edit.um.es/campusdigital/el-progreso-moral-no-ha-estado-al-mismo-nivel-que-el-progreso-tecnico-adela-cortina-nueva-doctora-honoris-causa-por-la-universidad-de-murcia/> [23/01/2021].

Viñals Blanco, Ana y Jaime Cuenca Amigo. 2016. “El rol del docente en la era digital” en Revista interuniversitaria de formación del profesorado, n³0 (2), 103-114. 


\section{Anexo}

\section{CUESTIONARIO INICIAL y FINAL:}

\section{Uso de TIC y herramientas de ciberseguridad en Estudios Franceses}

\section{1. ¿Qué apps conoces y/o usas?}

Alumno/a - A respuesta c. inicial: whatsapp, USCO, kindle, tik tok

Alumno/a - B respuesta c. inicial: calculadora, calendario (q vienen con el móvil) + redes sociales

Alumno/a - B respuesta c. final: conozco algunas apps que suben de redes sociales, además de Clash Royale y Améliorez votre français

Alumno/a - C respuesta c. inicial: whatsapp, Instagram, twitter, snapchat

Alumno/a - C respuesta c. final: spotify, groovepad, prezi, paint

Alumno/a - D respuesta c. inicial: gmail, word, netflix, youtube, play store

Alumno/a - D respuesta c. final: whatsapp, Instagram, power point, prezi, duolingo, babble, radio garden, drops, canva, youtube, triplingo, photoshop, adobe premiere pro

Alumno/a - E respuesta c. inicial: whatsapp, reverso, Instagram, youtube

Alumno/a - E respuesta c. final: whatsapp, biweager, shazam

Alumno/a - F respuesta c. inicial: whatsapp, Instagram

Alumno/a - F respuesta c. final: twitter, whatsapp, spotify, youtube

\section{2. ¿Qué redes sociales conoces y/o usas?}

Alumno/a - A respuesta c. inicial: Instagram, Facebook, twitter, Skype,

Alumno/a - B respuesta c. inicial: whatsapp, Instagram, twitter

Alumno/a - B respuesta c. final: whatsapp, facebook,Instagram, twitter, tik tok

Alumno/a - C respuesta c. inicial: whatsapp, Instagram, twitter

Alumno/a - C respuesta c. final: twitter, Instagram,

Alumno/a - D respuesta c. inicial: whatsapp, Instagram,

Alumno/a - D respuesta c. final: whatsapp, Instagram, twitter

Alumno/a - E respuesta c. inicial: Instagram, whatsapp,

Alumno/a - E respuesta c. final: twitter, facebook, Instagram

Alumno/a - F respuesta c. inicial: whatsapp

Alumno/a - F respuesta c. final: twitter, whatsapp 
3. ¿Qué webs conoces y/o usas?

Alumno/a - A respuesta c. inicial: Wikipedia, diccionario RAE

Alumno/a - B respuesta c. inicial: Dilo-un (Para ver series y películas)

Alumno/a - B respuesta c. final: DLE, wordreference

Alumno/a - C respuesta c. inicial: Wordreference, Prezi

Alumno/a - C respuesta c. final: Okodia

Alumno/a - D respuesta c. inicial: Gengo, Fivett

Alumno/a - D respuesta c. final: gengo, lyrics training, wordreference, open culture, TV Monde

Alumno/a - E respuesta c. inicial: Marca, RAE

Alumno/a - E respuesta c. final: Okodia, trip lingo, be funky

Alumno/a - F respuesta c. inicial: Google, youtube

Alumno/a - F respuesta c. final: Google,

\section{Menciona otras herramientas TIC que conozcas}

Alumno/a - A respuesta c. inicial: Edmondo, Reverso, youtube

Alumno/a - B respuesta c. inicial: Word, PDF, Excel, HotPotatoes, Zotero, CMaps

Alumno/a - B respuesta c. final: Tools, Prezi

Alumno/a - C respuesta c. inicial: Chaptools, Hotpotatoes

Alumno/a - C respuesta c. final: Word, hoja de cálculo, Paint

Alumno/a - D respuesta c. inicial: Hot Potatoes, Mapa Conceptual, Prezi, Excel

Alumno/a - D respuesta c. final: Excel, C Map Tools, Zotero

Alumno/a - E respuesta c. inicial: Prezi, Power Point, Photoshop

Alumno/a - E respuesta c. final: Prezi, Power Point, Kahoot

Alumno/a - F respuesta c. inicial: Prezi (un poco)

Alumno/a - F respuesta c. final: Prezi, canva, painter

\section{5. ¿Cuáles de ellas crees que se podrían aplicar a los Estudios Franceses (a conocer la} lengua, cultura, literatura francesas y francófonas)?

Alumno/a - A respuesta c. inicial: Diccionarios en línea, youtube, facebook

Alumno/a - B respuesta c. inicial: Word, PDF, Excel, HotPotatoes, Zotero, CMaps Tools

Alumno/a - B respuesta c. final: Wordreference, ya que te sirve para los significados, sinónimos, conjugación, ... y Prezi para hacer presentaciones y enseñar un tema

Alumno/a - C respuesta c. inicial: Chaptools (con él puedes hacer esquemas sobre cualquier tema),

Hot Potatoes (puedes hacer ejercicios didácticos con él) 
Alumno/a - C respuesta c. final: Cualquiera de ellas, porque con ellas utilizamos la lengua francesa y así se practica

Alumno/a - D respuesta c. inicial: Creo que todas las herramientas pueden aplicarse a los EF, sólo hay que tener

Alumno/a - D respuesta c. final: Creo que todas ellas pueden aplicarse a los EF si se posee creatividad

Alumno/a - E respuesta c. inicial: Prezi y Power Point son dos herramientas que pueden ayudarnos a la hora de hacer la explicación de un contenido determinado

Alumno/a - E respuesta c. final: Todas, aplicándolas de la manera correcta

Alumno/a - F respuesta c. inicial: Prezi

Alumno/a - F respuesta c. final: Todas, porque se pueden hacer presentaciones de diversos temas

\section{6. ¿Qué beneficios crees que tiene Internet (apps, redes sociales, webs, blogs,...)?}

Alumno/a - A respuesta c. inicial: -acceso a todo tipo de información.

-facilidad de comunicación entre personas que estén cerca o lejos.

Alumno/a - B respuesta c. inicial: Poder aprender y tener más materiales educativos

Alumno/a - B respuesta c. final: Nos permite buscar cualquier información enseguida

Alumno/a - C respuesta c. inicial: Puedes aprender cosas nuevas y obtener información para trabajos

Alumno/a - C respuesta c. final: Es un sitio en el que encuentras bastante información para trabajos y también hay noticias de actualidad. Tiene numerosas funciones que son útiles en nuestra vida diaria.

Alumno/a - D respuesta c. inicial: Facilita el acceso a la información

Alumno/a - D respuesta c. final: Permite que el conocimiento se difunda con facilidad y que todos podamos acceder a él.

Alumno/a - E respuesta c. inicial: Acceso a contenido casi ilimitado, rapidez, variedad

Alumno/a - E respuesta c. final: Muchísimas, es una fuente de información y contenido de todo tipo

Alumno/a - F respuesta c. inicial: Ampliar el conocimiento

Alumno/a - F respuesta c. final: Traer el conocimiento a la gente

\section{7. ¿Qué perjuicios crees que tiene Internet (apps, redes sociales, webs, blogs,...)?}

Alumno/a - A respuesta c. inicial: Suplantación de identidad, estafas, páginas web maliciosas 
Alumno/a - B respuesta c. inicial: Que no siempre se usa correctamente y puede acabar en dependencia o en ciber acoso

Alumno/a - B respuesta c. final: Puede ser adictivo y nos perjudica en todos los ámbitos

Alumno/a - C respuesta c. inicial: En internet hay muchas estafas que arrecian a mucha gente. También hay acoso

Alumno/a - C respuesta c. final: Internet puede ser también peligroso y perjudicial ya que hay gente que lo utiliza para hacer el mal. Esto también desemboca en el acoso.

Alumno/a - D respuesta c. inicial: No todo lo que está en internet está bien, hay que compartir la información con la de otras fuentes

Alumno/a - D respuesta c. final: La gente puede ser muy cruel e internet fomenta la toxicidad

Alumno/a - E respuesta c. inicial: Que es un sitio muy engañoso

Alumno/a - E respuesta c. final: En internet debes ser siempre políticamente correcto para no suscitar problemas para que las personas no se ofendan. Comienza a ser muy restrictivo.

Alumno/a - F respuesta c. inicial: Las "fake news"

Alumno/a - F respuesta c. final: Pone en riesgo los datos de la gente.

\section{8. ¿Qué opinas del ciberacoso, acoso virtual o "harcèlement"?}

Alumno/a - A respuesta c. inicial: A día de hoy es una amenaza a tener en cuenta y que se ven motivados por el anonimato que da internet

Alumno/a - B respuesta c. inicial: Se debería acabar. Hay que buscar nuevos métodos para eso

Alumno/a - B respuesta c. final: Es algo que debería acabarse y se debería hacer más acciones para su erradicación

Alumno/a - C respuesta c. inicial: Es algo que debería eliminarse, ya que se debe respetar a todo el mundo

Alumno/a - C respuesta c. final: No debería ocurrir ya que es algo muy peligroso

Alumno/a - D respuesta c. inicial: Creo que es una gran desventaja de Internet y que debe tratarse con dureza para que nadie sufra

Alumno/a - D respuesta c. final: Está muy mal y demuestra la cobardía de algunas personas

Alumno/a - E respuesta c. inicial: Es algo que se debería intentar tener más controlado

Alumno/a - E respuesta c. final: Debería estar mucho más penado

Alumno/a - F respuesta c. inicial: Es algo que hay que castigar (Miguel Ángel)

Alumno/a - F respuesta c. final: Que es algo muy malo 
9. ¿Sueles leer las condiciones de uso apps, redes sociales, webs, blogs, ... antes de aceptar las cookies, antes de instalarte una app, descargar una canción, un vídeo, una película, un programa...?

Alumno/a - A respuesta c. inicial: No

Alumno/a - B respuesta c. inicial: No

Alumno/a - B respuesta c. final: No

Alumno/a - C respuesta c. inicial: Lo leo por encima

Alumno/a - C respuesta c. final: Antes no lo hacía a menudo, pero ahora que conozco su funcionamiento y finalidad sí.

Alumno/a - D respuesta c. inicial: No

Alumno/a - D respuesta c. final: A veces

Alumno/a - E respuesta c. inicial: No

Alumno/a - E respuesta c. final: No

Alumno/a - F respuesta c. inicial: No

Alumno/a - F respuesta c. final: Ahora sí, le doy a preferencias y las ajusto

\section{0. ¿Crees que todo eso es gratis?}

Alumno/a - A respuesta c. inicial: Sí

Alumno/a - B respuesta c. inicial: No

Alumno/a - B respuesta c. final: No

Alumno/a - C respuesta c. inicial: No, porque seguramente dentro de la app intenten que pagues algo

Alumno/a - C respuesta c. final: No, porque aunque te descargues algo gratis, puede ser solo una tapadera y realmente te cobren algo, aunque sea poco.

Alumno/a - D respuesta c. inicial: No, en muchos casos esas páginas consiguen tus datos $\mathrm{u}$ obtienen beneficios con anuncios por ejemplo

Alumno/a - D respuesta c. final: No. Las empresas siempre obtienen algo a cambio. Nada es gratis.

Alumno/a - E respuesta c. inicial: No, al fin y al cabo siempre te solicitan permisos y accesos

Alumno/a - E respuesta c. final: No, es necesario dar datos que luego las empresas utilizan

Alumno/a - F respuesta c. inicial: No

Alumno/a - F respuesta c. final: No, nosotros somos el producto 
11. ¿Por qué crees que, si buscas, por ejemplo, unas zapatillas en la web un lunes, luego cada vez que enciendes el ordenador durante el resto de la semana o más te salen anuncios de zapatillas?

Alumno/a - A respuesta c. inicial: Usan nuestras búsquedas para personalizar la publicidad

Alumno/a - B respuesta c. inicial: Por las cookies. Una vez que buscas algo se te queda guardado y posteriormente te saldrán cosas similares

Alumno/a - B respuesta c. final: Por las cookies. Al aceptarlas, le estamos dando permiso para que usen nuestros datos.

Alumno/a - C respuesta c. inicial: Porque se va guardando en tu historial la página, y por lo tanto, te aparecen anuncios ara que las compres en otra página

Alumno/a - C respuesta c. final: Porque tu historial se guarda y cuando vuelves a encender el ordenador te salen esos anuncios para incitarte a comprar y gastarte dinero en esa tienda

Alumno/a - D respuesta c. inicial: Porque consiguen tu información y la usan para ofrecerte Alumno/a - D respuesta c. final: Las diferentes webs acceden a tu ubicación, contactos, etc., $\mathrm{y}$ te ofrecen productos para que termines comprando algo por insistencia.

Alumno/a - E respuesta c. inicial: Debido a algoritmos de búsqueda que nos relacionan con productos de nuestro interés

Alumno/a - E respuesta c. final: Es debido a las cookies que producen anuncios en relación a los productos que buscas

Alumno/a - F respuesta c. inicial: Porque tienen mis datos

Alumno/a - F respuesta c. final: Porque he aceptado recibir información

\section{2. ¿Conoces webs colaborativas como Change.org?}

Alumno/a - A respuesta c. inicial: no

Alumno/a - B respuesta c. inicial: Solo change.org

Alumno/a - B respuesta c. final: Solo change.org

Alumno/a - C respuesta c. inicial: Solo change.org

Alumno/a - C respuesta c. final: No, a parte de esa, ninguna más

Alumno/a - D respuesta c. inicial: Patreon

Alumno/a - D respuesta c. final: Okodia, Patreon

Alumno/a - E respuesta c. inicial: (No responde)

Alumno/a - E respuesta c. final: No

Alumno/a - F respuesta c. inicial: Sí, pero muy poco

Alumno/a - F respuesta c. final: Sí, pero es un timo porque puedes firmar varias veces 


\section{3. ¿Conoces Youtubers, Influencers, Streamers?}

Alumno/a - A respuesta c. inicial: Sí. (No da nombres)

Alumno/a - B respuesta c. inicial: Malcaide- jugador de Clash Royale

Dulceida- youtuber de varios temas (ropa, trabajo)

Elsa Ruiz- youtuber y monologuista sobre temas LGT-

BI, en especial sobre el colectivo trans

Air Magno (RAP)

Dropkicks Murphys (Música irlandesa)

Otros artistas de RAP: SFDK, Morodo, Veneno Crew

Alumno/a - C respuesta c. inicial: Conozco algunos influencers de moda en Instagram

Alumno/a - D respuesta c. inicial: Meimuna, Stromae, Alma, Francis Cabrel, Indila, Soprano, Indochine.

Kevin Bordi (es un jugador profesional de ajedrez)

Alumno/a - D respuesta c. final: Dulceida, Andyygmes, Dein_Sprochcoach, Superholly, Nutisa, Tom Merrich, Dad, Mariaspeakenglish

Alumno/a - E respuesta c. inicial: Stick, Zetassj

Alumno/a - E respuesta c. final: Ibai, Rubius, Mario Dualle, Stick

Alumno/a - F respuesta c. inicial: Juan Ramón Rallo (Economista)

Javier Milei (Economista)

Rubén Gisbert (abogado)

Rama Gallardo (Activista)

Libertad TV (Política)

Jesús Huerta de Soto (Catedrático)

Alumno/a - F respuesta c. final: Rallo, Milei, Gisbert + Rama,

14. ¿Conoces apps, webs, instituciones, organismos relacionados con la protección de datos?

Alumno/a - A respuesta c. inicial: No

Alumno/a - B respuesta c. inicial: No

Alumno/a - B respuesta c. final: Agencia de protección de Datos, Lista Negra

Alumno/a - C respuesta c. inicial: No

Alumno/a - C respuesta c. final: Sí

Alumno/a - D respuesta c. inicial: No

Alumno/a - D respuesta c. final: Lista Robinson, CNIL, CEDRO

Alumno/a - E respuesta c. inicial: No

Alumno/a - E respuesta c. inicial: AEPD, CEPD

Alumno/a - F respuesta c. inicial: No

Alumno/a - F respuesta c. final: Sí, la agencia de protección de datos española, la francófona 
